\title{
PERSPECTIVAS DEL PROYECTO QHAPAQ ÑAN EN TORNO AL REGISTRO DE LA RED VIAL INCA: PROPUESTAS DE SU SECTORIZACIÓN Y NOMENCLATURA
}

\author{
PERSPECTIVES OF THE QHAPAQ NAN PROJECT ON THE REGISTRY \\ OF THE INCA ROAD NETWORK: PROPOSALS FOR SECTORIZATION \\ AND NOMENCLATURE
}

Alfredo Bar Esquivel ${ }^{A}$

Desde el año 2003, el Proyecto Qhapaq Ñan-Perú ha realizado diversos trabajos de investigación y registro de la red de Caminos Incas extendida en el territorio peruano con el fin de promover su conocimiento, conservación y puesta en uso social. La experiencia obtenida en el desarrollo de estas actividades ha permitido generar una propuesta de nomenclatura para los caminos basada en su originalidad y continuidad de uso. El presente texto hace una breve reseña de las diferentes etapas del trabajo de campo llevadas a cabo y la problemática que han llevado a la elaboración de nuevas definiciones y el rediseño de las técnicas de recolección de datos, con la elaboración de nuevos instrumentos de registro que buscan contribuir con el entendimiento del estado situacional de la red vial inca.

Palabras clave: registro, red vial, tramo, sección.

Since 2003, the Qhapaq Nan-Peru Project has carried out research and registration of the Inca road network throughout the Peruvian territory in order to promote its knowledge, conservation and social use. Experience obtained during the development of these activities led to a proposal of nomenclature for Inca roads based upon originality and continuity of use. The present text briefly outlines the stages of the fieldwork carried out here, the problems involved in the making of new definitions, as well as the redesign of data collection techniques, including the development of new registration tools intended to contribute to the understanding of the state of the Inca road network.

Keywords: registry, road network, road section.

\section{INTRODUCCIÓN}

Desde hace casi quinientos años, diversos autores han descrito en sus obras las secciones que forman parte del Qhapaq Ñan. Cronistas como Miguel de Estete (1533), Pedro Cieza de León (1553), Pedro Sarmiento de Gamboa (1572), Martín de Murúa (1590) y Felipe Guaman Poma de Ayala (1615), por citar solo algunos, dan cuenta -en sus crónicas- de la admiración que les causaran los Caminos del Inca en su tránsito durante el proceso de conquista, o bien durante labores de índole eclesiástica o administrativa. Aunque sus escritos suelen estar marcados por un juicio historicista en favor del régimen colonial, constituyeron un primer acercamiento al estudio de caminos. Posteriormente, en el siglo XIX viajeros como Jacob Tschudi (1838-1842), Antonio Raimondi (1851-1869), Charles Winner (1875-1877) y Ernst Middendorf (1876-1888) tomaron la documentación etnohistórica como base para el conocimiento de la red vial inca y elaboraron también, luego de recorrer los caminos, los primeros mapas del Qhapaq Ñan. Esto fue posible gracias al auspicio del gobierno peruano, preocupado de impulsar el intercambio de productos entre la costa y la sierra a través del diseño de alternativas

\footnotetext{
A Alfredo Bar Esquivel, Arqueólogo de la Coordinación de Investigación y Registro de la Red Vial Inca. Proyecto Qhapaq Ñan, Perú. R. Orellano 190, Santa Luzmila, Lima 7, Perú, email: abarsel@hotmail.com
} 
viales que brindasen un mayor flujo de productos para su exportación (véase Bar 2013).

En el siglo xx, el interés por el Qhapaq Ñan llevó a nuevos investigadores a retomar la revisión de las crónicas y otros documentos redactados por funcionarios coloniales, como ordenanzas y visitas, con el fin de recorrer los caminos (aunque no en toda su extensión) y elaborar mapas más detallados de la red vial inca. Tal es el caso del ingeniero peruano Alberto Regal (1936, 1972), el padre estadounidense León Strube Erdmann (1963) y el periodista prusiano Víctor von Hagen (1977), quienes establecieron las bases del estudio metódico de la red vial inca, que luego servirían en la sistematización del estudio científico realizado por John Hyslop (1992) y otros autores como R. Raffino cuyo trabajo de sistematización de caminos y sitios incas, del Noreste Argentino, Los Inkas del Kollasuyo, fue publicado previamente en 1981.

En los últimos años, el estudio de caminos prehispánicos en el ámbito peruano se ha desarrollado dentro del marco de desarrollo del Proyecto Qhapaq Nan (PQÑ) desde su creación el año 2001 a raíz de la publicación del Decreto Supremo $\mathrm{N}^{\circ} 31$-2001-ED que declara de interés nacional la investigación, identificación, registro, protección, conservación y puesta en valor de la red de caminos existentes en el imperio incaico dentro del territorio nacional, dando preferente atención al Gran Camino Inca, conocido como Qhapaq Nan. En atención a este decreto el Perú inició el registro del Qhapaq Ñan y, posteriormente, a mediados del año 2002, suscribió un acta de compromiso para fomentar acciones conjuntas a favor del mismo con los países de Argentina, Bolivia, Colombia, Chile y Ecuador, durante la Reunión del Patrimonio Mundial llevada a cabo en Montevideo (Uruguay). Ese mismo año el gobierno peruano propuso inscribir al Qhapaq Nan en la lista tentativa del Patrimonio Mundial ante la UnEsCo (Figueroa 2009: 11). Esta propuesta fue acogida, por los países mencionados, en la XVII Cumbre del Grupo de Río (2003) realizada en Cuzco, y derivó en la creación del PQÑ-Camino Principal Andino (Bákula 2009: 4) cuyo objetivo era lograr la inclusión de la red vial inca, y de los sitios asociados a esta, dentro de la Lista del Patrimonio Mundial, meta alcanzada en junio del 2014 y que, en el caso peruano, implicó la declaratoria de seis tramos y ochenta y dos sitios arqueológicos.

En este contexto, a fin de colegir los datos obtenidos en las diversas campañas, el PQÑ-Sede Nacional, del Ministerio de Cultura, Perú, viene realizando desde el 2012 la revisión de los informes presentados en las distintas temporadas y ha notado, en principio, su dependencia del criterio subjetivo de los arqueólogos, aun cuando se había consensuado un instructivo para el manejo de información entre los países que integraban el PQÑ-Camino Principal Andino, en vías de su nominación como Patrimonio Mundial. Este hecho, sumado a la profusa ejecución de trabajos, dentro de la llamada arqueología de contrato, expuso la necesidad de reestructurar los lineamientos técnicos del acopio y procesamiento de datos a fin de integrarlos en una sola base informática, independientemente de su origen.

En el siguiente texto, realizamos un recuento de los criterios empleados por el PQÑ en el registro de caminos, y centramos la discusión en las propuestas de sectorización y nomenclatura de transectos y su categorización.

\section{ESTRATEGIAS DEL INSTITUTO NACIONAL DE CULTURA (INC) PARA EL REGISTRO DEL QHAPAQ ÑAN}

Tomando como punto de partida el Decreto Supremo $\mathrm{N}^{\circ}$ 31-2001-ED, el Estado peruano emprendió la ambiciosa labor de inventariar la vasta red de Caminos Incas y sitios arqueológicos asociados, encomendando esta misión al Instituto Nacional de Cultura (actual Ministerio de Cultura) que por medio del PQÑ realizó varias temporadas de campo (2003, 2004 y 2008).

Las primeras pautas de registro fueron establecidas en el 2003 en el marco del Proyecto de Levantamiento de Información del Sistema Vial Inca (PLISVI), y se organizó su recorrido en torno a cuatro macro regiones (norte, centro, centro-sur y sur) que congregaban regiones específicas sobre la base de su realidad geopolítica y facilitaban el control logístico del trabajo de campo considerando también el estudio de comunidades modernas y su diagnóstico situacional para conocer cómo el Qhapaq Ñan se integra con el desarrollo social del Perú.

Entre otras, el componente arqueológico del PQÑ tenía como funciones: a) registrar y evaluar el estado de conservación de las estructuras arqueológicas del sistema vial inca; b) generar mapas temáticos usando un Sistema de Información Geográfica (SIG), a partir del inventario de tramos y sitios; c) generar fichas catastrales de los tramos y sitios asociados al sistema vial inca; d) promover, concebir y ejecutar planimetrías 
para el estudio de tramos de camino y sitios asociados a partir de los datos registrados en el levantamiento de la información; e) promover, concebir y ejecutar proyectos de investigación arqueológica a partir de los datos registrados en el levantamiento de información.

No obstante, el accionar técnico no fue uniforme en todo el ámbito de intervención, pues mientras en las macro regiones norte, centro y sur se realizaron prospecciones en torno al eje lineal de los caminos, en la macro región centro-sur estas se organizaron por áreas geográficas. Asimismo, aunque la información recibida de los equipos de campo (fichas, puntos tomados con navegador GPs en Datum WGS84, informes, etc.) pasó por un proceso de verificación y depuración con respecto a las descripciones del PLisvi para la generación de una base de datos única, esta mostraba marcadas diferencias entre el dato original y su proyección en el sIG, por ello los caminos no podían ser fácilmente identificados dentro del ámbito de acopio del dato por los diversos investigadores o arqueólogos de las sedes regionales del INC. Además, los caminos eran representados como una sola unidad sin precisar su originalidad, reconstrucción o pérdida total, debido, por ejemplo, a la superposición de obras viales, lo que llevó a cuantificar un total de $8.194 \mathrm{~km}$ de caminos recorridos el 2003, a los que se vinieron a sumar $3.132,512 \mathrm{~km}$ el 2004, entre tramos de camino reconocidos y su trazo. ${ }^{1}$

La clasificación de transectos fue explicada en la versión digital de los informes de campaña (CD de difusión por macro región) del siguiente modo: por un lado, camino reconocido es aquel que tiene sus elementos constitutivos (calzada de arena, tierra, piedra, sobreelevada de tierra o piedra, muros laterales de contención superior y/o inferior, alineamientos laterales de piedras, escalinata, etc.); obras de infraestructura vial (puentes, canaletas de drenaje, etc.), en regular o buen estado de conservación. Es decir, que los agentes de deterioro no lo han afectado en gran medida. En este sentido, el camino es claramente identificable de modo tal que se puede seguir por él ya que se encuentra bien definido. Por otro, trazo de camino es donde no se encuentra camino, pero es posible ver aún sus restos, es decir, sus elementos constitutivos, la arquitectura vial presente en él si la tuviera (Instituto Nacional de Cultura 2005: 10).

A fin de contar con un mismo criterio de operación, en el 2006 se establecieron pautas de registro técnico con mayor detalle, en consenso con los países que integraban el PQÑ-Camino Principal Andino. La nueva metodología, plasmada en el "Instructivo para el manejo de las fichas de caminos", permitía mantener el uso de las macro regiones (fig. 1) en las que exclusivamente debería seguirse el eje lineal de los caminos y efectuarse el reconocimiento de sitios asociados a estos en un rango de $200 \mathrm{~m}$ a partir de sus bordes; además de ello, conceptualizaba el tramo como la proyección mayor a $100 \mathrm{~km}$ de una ruta, en contraste a la distancias recorridas los años 2003 y 2004. ${ }^{3}$ Una siguiente división podía darse en subtramos (distancias mayores a $10 \mathrm{~km}$ ) y segmentos (distancias menores a $10 \mathrm{~km}$ ) que eran categorizados de acuerdo con su visibilidad y su estado de conservación como: camino reconocido, trazo de camino y posible ruta para distinguir entre la extensión real de un camino de origen arqueológico y otro que hubiese perdido esta condición debido a las continuas remodelaciones y/o reconstrucciones hechas por las comunidades de la zona geográfica en la que se emplaza.

El implemento de una "posible ruta" definía aquellas proyecciones donde no se reconocía evidencia arqueológica, debido a la superposición de alguna trocha carrozable, carretera, autopista o las modificaciones por obras civiles o de otra naturaleza. Casaverde y López (2010: 95) resaltan la dificultad de determinar esta categoría cuando señalan: "La posible ruta se puede inferir a partir del análisis y evaluación de las condiciones del medio físico en las que se pudo ubicar el camino y, con algo de suerte, gracias al hallazgo de restos del camino bajo los escombros arrojados al momento de abrir una carretera, por ejemplo".

Si bien el planteamiento de una nueva metodología era auspicioso, finalmente su aplicación dependía del criterio de cada profesional, ya que en general la selección de "tramos" estaba organizada sobre la base del conocimiento de caminos que no necesariamente estaban integrados entre sí, por lo que no había correspondencia entre la longitud señalada en el instructivo de registro y el registro de campo. Además, la nomenclatura de tramos se realizaba de acuerdo a los puntos inicial y final del recorrido, lo que no reflejaba necesariamente el sentido de articulación del camino con la red vial inca. Por último, no había clara distinción sobre la originalidad de las secciones de caminos y las transformaciones ocurridas por su tránsito continuo.

Al estar supeditados a las apreciaciones inmediatas de los investigadores, los datos obtenidos en campo pocas veces eran correlacionados con aquellos provenientes de la arqueología de contrato, que entre el 2003 y el 


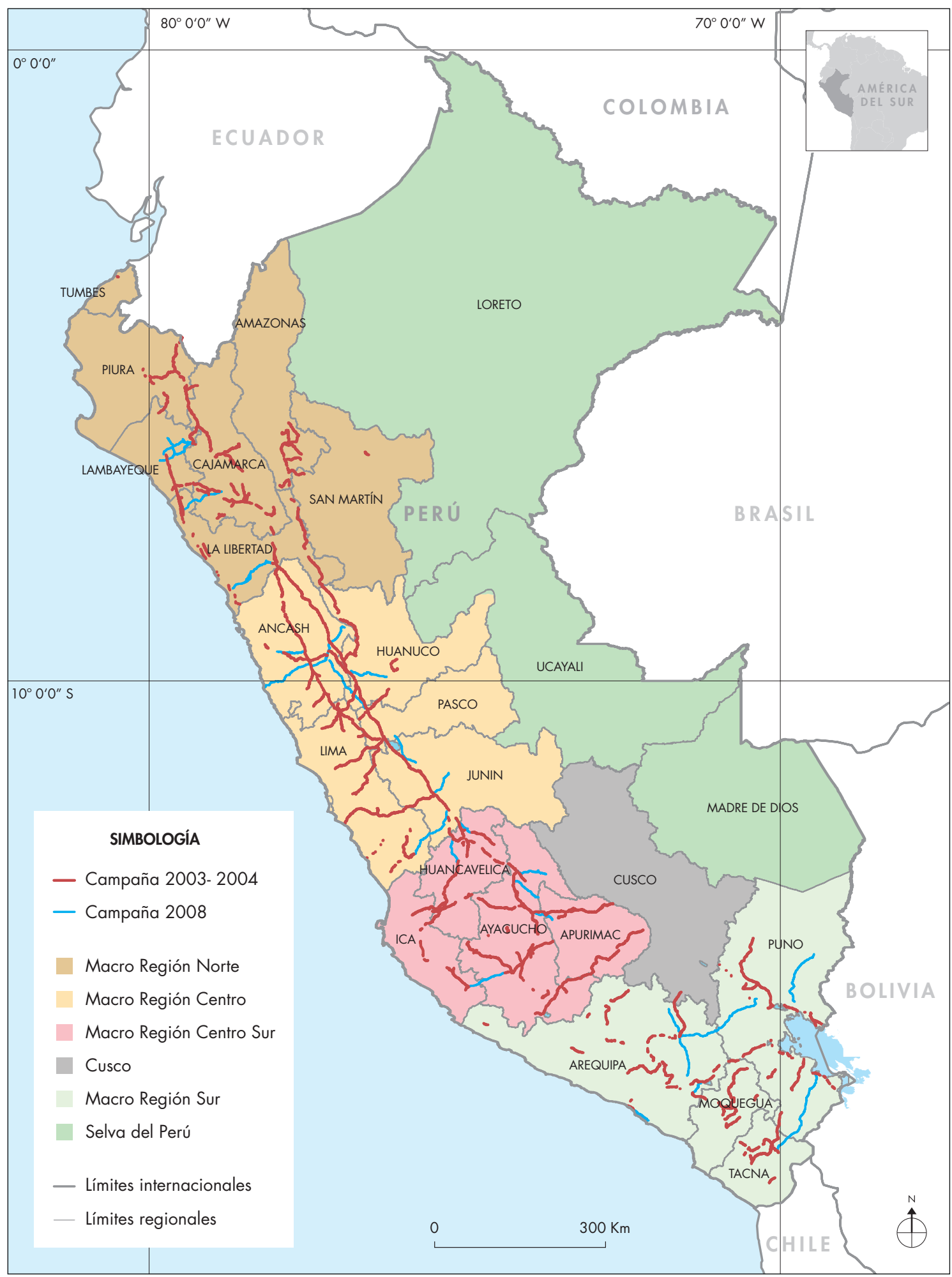

Figura 1. Red de caminos identificados por macro región, entre los años 2003-2008. Figure 1. Network of identified roads by macroregion, between 2003 and 2008. 
2009 superaban ampliamente a los de investigación y proporcionaban "oportunidades únicas para el registro y recuperación de información arqueológica que, de otra manera, permanecería ignorada o se perdería irremediablemente" (Ríos 2014: 170). Esta situación podía derivar en una documentación reiterativa, como en el caso del Camino Inca que se emplaza en la quebrada de Chuspiragra, distrito de Huallanca, región Ancash, y que fuera reconocido previamente por dos proyectos de evaluación arqueológica y luego denominado por el PQÑ como "sección Chuspiragra-Hornillos" (Bar 2008). ${ }^{4}$

En el mismo contexto, secciones del camino longitudinal de la sierra fueron objeto de un doble reconocimiento. El ejemplo más palpable se dio entre las localidades de Pazos y Marcavalle, ubicadas en las regiones de Huancavelica y Junín respectivamente. Hacia el 2003, esta sección fue registrada como parte del tramo Acostambo-Jauja (orientación sur-norte) y el 2008 como parte del tramo Marcavalle-Pampas (orientación norte-sur) (Varela 2003; Bernabé 2008).

Actualmente, la Coordinación de Investigación y Registro de la Red Vial Inca (IRRVI), después de analizar los alcances de los trabajos realizados, ha considerado replantear las estrategias de recopilación de datos y su correlación con toda la información de campo existente -indistintamente de su procedencia- a fin de crear una sola Geodatabase que pueda ser aprovechada por el PQÑ, las diversas áreas técnicas del Ministerio de Cultura y los profesionales e instituciones que centran su estudio en la red vial inca.

\section{SECTORIZACIÓN DE LA RED VIAL INCA}

En diciembre del 2013, el PQÑ publicó la Guía de Identificación y Registro del Qhapaq Nan, instructivo para la recolección de datos y diagnóstico situacional de los Caminos Incas. Esta publicación, que tuvo aceptable acogida, viene siendo revisada y "actualizada" con base en los proyectos de investigación desarrollados por la Coordinación IRRVI y las supervisiones conjuntas con las diversas direcciones del Ministerio de Cultura, en aquellos transectos identificados en evaluaciones arqueológicas.

Como se indica en dicha guía, "[...] los caminos están compuestos no solo por diversos elementos de infraestructura vial sino también por diferentes tipos de establecimientos que en conjunto integran un sistema vial localizado en distintos ámbitos geográficos que han influido en su planificación y construcción. Estos factores también deben ser considerados en el registro y en la posterior interpretación de las evidencias arqueológicas" (Ministerio de Cultura 2013: 71).

Entendiendo que los caminos forman parte de un paisaje integrado al quehacer social y cultural de los pueblos que los transitan, su estudio debe extrapolar diversos factores como la distancia entre asentamientos, tiempo de viaje y facilidad del recorrido entre ellos, además de su densidad demográfica, etc. (Castro et al. 2004; Topic \& Topic 2014). Así, por más tediosa que parezca la cuantificación de detalles o cambios estructurales existentes, esto debe coadyuvar en el reconocimiento de cánones constructivos que expliquen su origen, uso y función en relación con cada época cultural arqueológica.

Para un primer acercamiento al estudio de la red vial inca deben emplearse las representaciones de nodo (sitios) y eje lineal (caminos) como atributos gráficos y la información asociada a estos (como atributos no gráficos) que puedan ser integrados a un Sistema de Información Geográfica (SIG). Por ello, se cree conveniente mantener la categorización de transectos, pero definiéndolos en torno a sus nodos de conexión, antes que su extensión lineal, y contemplando en el estudio de tramos las secciones de camino que no forman parte de su eje lineal pero sí de la red articulada en torno a este, sea en cuencas hidrográficas o divisiones políticas referenciales (históricas o actuales), por lo que la nomenclatura a emplearse debe estar acorde con los nodos que forman parte de la red vial, en preferencia sobre los puntos que demarcan la extensión del registro de campo, que debe realizar además una nueva evaluación de los caminos en torno a su visibilidad (originalidad y transformaciones dadas por su uso).

Asimismo, bajo este criterio se proponen las siguientes unidades de sectorización: a) tramo: el trayecto cuya distancia promedia, o supera, los $100 \mathrm{~km}$; b) subtramo: trayecto variable entre 10 y $100 \mathrm{~km}$; c) sección: trayecto menor que varía entre 1 y $10 \mathrm{~km}$; d) segmento: la unidad mínima de medición menor a 1 km (fig. 2).

Aunque pareciera repetirse el criterio del 2008, no se establecen unidades fijas de medida, ya que cada categoría varía o depende, además de la geomorfología en la cual se emplaza el camino, de los nodos que definen su extensión y trayectoria, ya sean sitios arqueológicos de relativa importancia, localidades modernas o accidentes geográficos de significativa particularidad. 


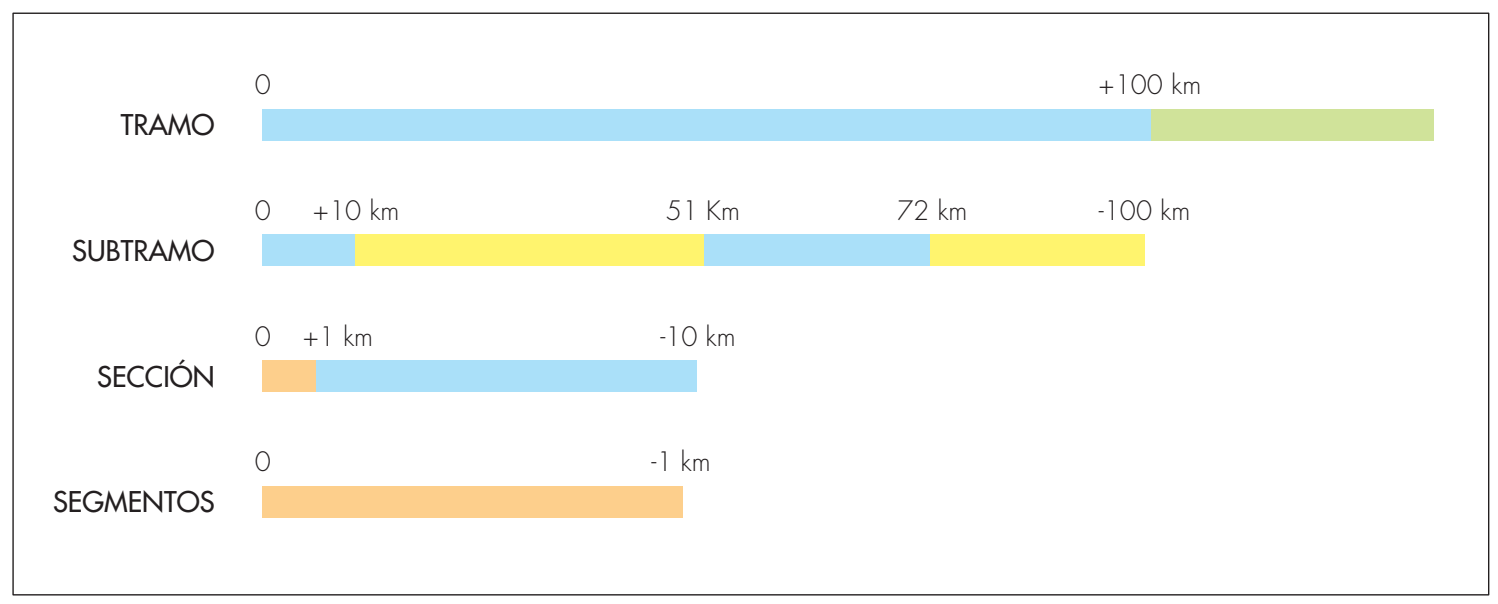

Figura 2. Categorización de transectos de caminos del Qhapaq Nan. Figure 2. Categorization of sections of the Qhapaq Nan roads.

El tramo, visto en esta perspectiva, no define la distancia métrica en sí misma, como se hace en la segmentación de una obra vial moderna, sino la proyección de un eje lineal que pueda o no superar los $100 \mathrm{~km}$. Su uso se orienta a la descripción de una ruta específica y su entorno geográfico, comprendidos entre dos sitios de relativa preeminencia (nodos primarios). ${ }^{5}$ Tal es el caso, por ejemplo, de la ruta -de más de $320 \mathrm{~km}$ - que se sigue en el camino longitudinal de la sierra, entre los asentamientos incas de Huánuco Pampa y Huamachuco, centros administrativos ubicados en los actuales departamentos de Huánuco y La Libertad, tipificados en la bibliografía histórica como "cabezas de provincia”. Esto mismo ocurre con las rutas transversales que conectan localidades modernas, superpuestas a antiguos centros administrativos, como es el caso de las ciudades de Huaraz y Casma, localizadas en las regiones centrales de la sierra y costa peruanas, respectivamente, $y$ distanciadas entre sí por unos $90 \mathrm{~km} .{ }^{6}$

Para el caso de los subtramos, su uso es factible solo cuando la anterior categoría ha sido ya establecida, y necesariamente al interior de ella. La sectorización se corresponde con la distancia existente -no siempre equidistante- entre los sitios arqueológicos, o las localidades asentadas sobre estos, que han servido como nodos secundarios o "intermedios" en relación con el conjunto existente en la proyección de un mismo eje vial. Estos sitios pueden ser localizados mediante el estudio de diversas fuentes históricas -la ordenanza de tambos de Vaca de Castro (1909 [1543]), por ejemplo-, así como por el estudio arqueológico que contempla la integración al entorno geográfico. Dichos nodos son aquellos asentamientos que controlan el flujo de productos o poblaciones entre los nodos primarios. ${ }^{7}$ Un ejemplo práctico de esta sectorización puede verse en la división del Tramo Xauxa-Pachacamac, que en su extensión lineal de 217,70 km cuenta con cuatro subtramos: a) Hatun Xauxa-Julca Tambo $(46,81 \mathrm{~km})$; b) Julca Tambo-Huarochirí (65,49 km); c) HuarochiríSisicaya (59,26 km); d) Sisicaya-Pachacamac (46,14 km).

La sección y el segmento como integrantes de las unidades mayores -subtramo y tramo- se asocian a la extensión del camino definida tanto por el relieve y la superficie del terreno, como por su estado de conservación. Lo resaltante de una sección es su proyección entre nodos de menor rango, que pueden ser: sitios arqueológicos, localidades modernas, puntos de intersección vial y accidentes geográficos que demarcan cambios en su traza o el paso entre regiones propiamente dichas. Pueden considerarse también aquellos accidentes geográficos que por su geomorfología son elementos preeminentes sobre el paisaje o han adquirido un carácter especial en la concepción de las localidades que se asientan en torno a estos. Tenemos como ejemplo la sección: Iglesia Cochamarca-Tablachaca o Camino Inca de Cochamarca $(5,73 \mathrm{~km})$, que forma parte del subtramo Upamayo-Corohogo y que en toda su proyección, en el llano altoandino de la región Pasco, mantiene una traza lineal recta y bordes simples formados por surcos excavados en el terreno. ${ }^{8}$

Por su parte, el segmento se emplea por temas de operatividad que, aunque arbitraria, depende de las 
características del camino y su estado de conservación, y no de los nodos de conexión. La recurrencia de elementos arquitectónicos, como los drenes subyacentes o la falta de estos, en una calzada empedrada, por ejemplo, puede determinar su longitud total, la misma que es relativamente corta. Tomando en cuenta los proyectos de puesta en valor que prevén acciones de restauración, el segmento puede ser subdividido en unidades menores o "componentes" a fin de maximizar el diagnóstico de su estado de conservación. Los criterios de dicha división pueden ser desarrollados en la competencia de cada proyecto.

Hasta aquí el criterio de sectorización observa una jerarquización de nodos, situación que no le era ajena al Estado Inca al momento de ordenar su propia red vial y los centros administrativos que vinculaba; lo cual se identifica en las nominaciones que Guamán Poma (2008 [1615]: 34) hace de estos como: Tambo real, Tambo, Tambillo, etc., en referencia a su importancia dentro del aparato estatal. Aunque sometida a discusión hoy en día, el empleo de la categorización señalada, obra como un medio instrumental que facilita el registro de campo. La interpretación de un sitio arqueológico como centro administrativo, o tambo, solo puede darse tras comparar su particularidad arquitectónica con aquellas características físicas reconocidas para los sitios incas (Kendall 1976; Gasparini \& Margolies 1977; Hyslop 1992) y su asociación al entorno y sitios locales. ${ }^{9}$ Bajo esta propuesta, el uso o interpretación de un sitio arqueológico como nodo primario o secundario, en contraste con los aspectos geográficos y lugares sagrados, se hace a razón del origen del Qhapaq Nan cuya configuración mantenía un orden político y administrativo. ${ }^{10}$ Los sitios sagrados, montañas y adoratorios de altura son generalmente anteriores a la expansión del imperio inca; su origen no se debe al Qhapaq Nan y su integración al mismo finalmente formaba parte de estrategias políticas (Vitry 2007; Astuhuamán 2010). No obstante, la correlación que se haga de cada uno de estos, cualquiera sea su nominación, debe discernir su interacción, como sucede con el Tramo Xauxa-Pachacamac, que integra un centro administrativo inca y un centro religioso panandino de origen anterior al Tahuantinsuyu, que finalmente fue incorporado políticamente a este.

\section{NOMENCLATURA DE LA RED VIAL INCA}

Como se expone en distintos estudios sobre redes de camino, estas evolucionan de modo dinámico, reflejando en su diseño el flujo del tránsito, así como los eventos políticos y económicos de los nodos que la componen "y, en menor grado, la manipulación de las rutas por entidades políticas más pequeñas de las zonas de transición a través de las cuales discurre la ruta" (Topic \& Topic 2014: 57). Como consecuencia, consideramos que el estudio de la red vial inca debe contemplar el principio de jerarquización entre los diversos centros administrativos del Qhapaq Nan, ya que su construcción se orientó a servir como un complejo sistema administrativo, de transportes y de comunicaciones, así como un medio para delimitar las cuatro regiones del Imperio Inca (Hyslop 1992: 31), por lo cual su denominación debe seguir también un sentido radial teniendo a la ciudad de Cuzco como punto origen a partir del cual se produce su extensión:

Desta plaza [del Cuzco] salían cuatro caminos reales; en el que llamaban Chinchasuyo se camina a las tierras de los llanos con toda la serranía, hasta las provincias de Quito y Pasto; por el segundo camino, que nombran Condesuyo, entran las provincias que son subjetas a esta ciudad y a la de Arequipa. Por el tercero camino real, que tiene por nombre Andesuyo, se va a las provincias que caen en las faldas de los Andes, y algunos pueblos que están pasada la cordillera. En el último camino destos que dicen Collasuyo entran las provincias que llegan hasta Chile. De manera que, como en España los antiguos hacían división de toda ella por las provincias, así estos indios, para contar las que había en tierra tan grande, lo entendían por sus caminos (Cieza de León 1995 [1553]: 258; fol. 118).

Todo el Reino estaba dividido en cuatro partes, que llamaban Tahuantinsuyo, que eran Chinchasuyo, Collasuyo, Andesuyo, Condesuyo, conforme a cuatro caminos que salen del Cuzco, donde era la Corte, y se juntaban en juntas generales. Estos caminos y provincias que les corresponden están a las cuatro esquinas del mundo, Collasuyo, al Sur, Chinchasuyo, al Norte, Condesuyo, al Poniente, Andesuyo, al Levante (Acosta 1954 [1590]: 418).

Como podrá verse en la nueva Guía de identificación y registro del Qhapaq Nan, la denominación de los tramos debe darse de la siguiente manera:

- Tramos que se ubican hacia al norte de Cusco se denominarán de sur a norte, como por ejemplo: Huánuco Pampa-Huamachuco, Aypate-Las Pircas, etc. 
- Tramos que se ubican al oeste de Cusco o del Camino Longitudinal de la Sierra se denominarán de este a oeste, como por ejemplo: Cusco-Vilcashuamán, Vilcashuamán-Pisco (Tambo Colorado), XauxaPachacamac, etc.

- Tramos que se ubican al este de Cusco o del Camino Longitudinal de la Sierra, como los que se dirigen hacia la Selva, se denominarán de oeste a este. Por ejemplo: Cusco-Paucartambo, Ninacaca-Huancabamba, etc.

- Tramos que se ubican al sur de Cusco se denominarán de norte a sur, como por ejemplo: Cusco-Desaguadero, Cusco-Arequipa, etc.

Solo para el caso del Camino Longitudinal de la Costa o de los Llanos se recomienda seguir una orientación norte-sur, en atención a diferenciarlo del Camino Longitudinal de la Sierra (fig. 3), ya que su construcción habría empezado en la región de Tumbes bajo órdenes del Inca Túpac Yupanqui y su articulación final solo fue posible, según relatos de los cronistas europeos, luego que el Inca Huayna Capac, consolidara la conquista de Quito:

abaxó a lo de Túnbez y fue honradamente recibido por los naturales, a quienes Topa Ynga mostró mucho amor; y luego se puso del traje quellos usa[va]n para más contentarles y alabó a los prencipales el querer sin guerra tomalle por señor, [y] prometió de los tener y estimar como a hijos propios suyos. Ellos, contentos con oyr sus buenas palabras y manera con que se tratava, dieron la obediencia con onestas condiciones y permitieron quedar entre ellos governadores y hazer edificios; [...] Saliendo de aquel valle caminó el rey Ynga por lo más de la costa, yendo haziendo el camino real tan grande y hermoso como oy parece lo que dél ha quedado (Cieza de León 1996 [1553]: 169; fol. 70).

cuando otra vez el mismo Guaynacaba quiso volver a visitar la provincia de Quito, a que era muy aficionado por haberla el conquistado, torno por los llanos, y los indios le hicieron en ellos otro camino de casi tanta dificultad como el de la sierra (Agustín de Zárate, en Garcilaso 2007 [1604]: 800).

En atención a este hecho, los tramos que integran el Camino Longitudinal de la Costa podrán ser denominados en orientación norte-sur, como por ejemplo: TumbesMotupe, Chincha (La Centinela)-Nazca, Nazca-Atico, Arequipa-Sama, etc.

Finalmente, la orientación específica de cada tramo -longitudinal o transversal- debe servir también en la descripción técnica de las secciones que lo conforman. En la práctica, la descripción del tramo Tumbes-Motupe, por ejemplo, iniciará con la primera sección hallada en Tumbes y finalizará con aquella que se encuentre en
Motupe. En el caso de los segmentos, al ser divisiones menores, su nomenclatura puede consignarse mediante el uso de numerales que seguirán también el sentido de orientación de la sección en la cual están insertos.

\section{Redes alternas y la nomenclatura de sus secciones}

La proliferación de sitios fuera de un único eje vial, así como la presencia de sitios arqueológicos aislados geográficamente, por lo general origina la ramificación de caminos que funcionan en torno al eje lineal de mayor longitud. Como consecuencia, un tramo puede adscribir el entorno geográfico en el que se emplaza, aun cuando este sea de gran extensión (por ejemplo, una cuenca hidrográfica), siempre y cuando logre vincular sus nodos, sean sitios arqueológicos o geografía histórica, con la red vial inca. Nuevamente el tramo Xauxa-Pachacamac permite ejemplificar lo dicho, pues una vez que traspasa la cordillera del Pariacaca, hacia el oeste, se extiende en un valle de amplitud reducida (en la cuenca del río Lurín) y sirve como eje articulador de los caminos que comunican los diversos asentamientos arqueológicos ubicados tanto en el cono de deyección del valle bajo como en las quebradas escarpadas del valle medio (fig. 4).

El principio de nomenclatura de las secciones que componen estas redes puede regirse también al orden establecido previamente, pero partiendo siempre del eje lineal del tramo mayor. En el caso de secciones de corta extensión, o de sendas anteriores a la red vial inca, estas deben nominarse sin uso de la categorización propuesta, teniendo como criterio la jerarquización (por área y monumentalidad) de los sitios que conectan; esto si aún no se ha podido establecer el orden u origen de su sistema vial o, también, teniendo como indicadores los referentes geográficos inmediatos. Solo cuando los caminos parten de un punto específico hacia otra cuenca o región, y llegan también a un sitio arqueológico, pueden ser considerados como unidades Tramo indistintamente de su longitud.

\section{DEFINICIONES EN TORNO A LA VISIBILIDAD DE CAMINOS}

La experiencia adquirida por la coordinación IRRVI en el desarrollo de proyectos de investigación y las funciones de una institución gubernamental, como lo 


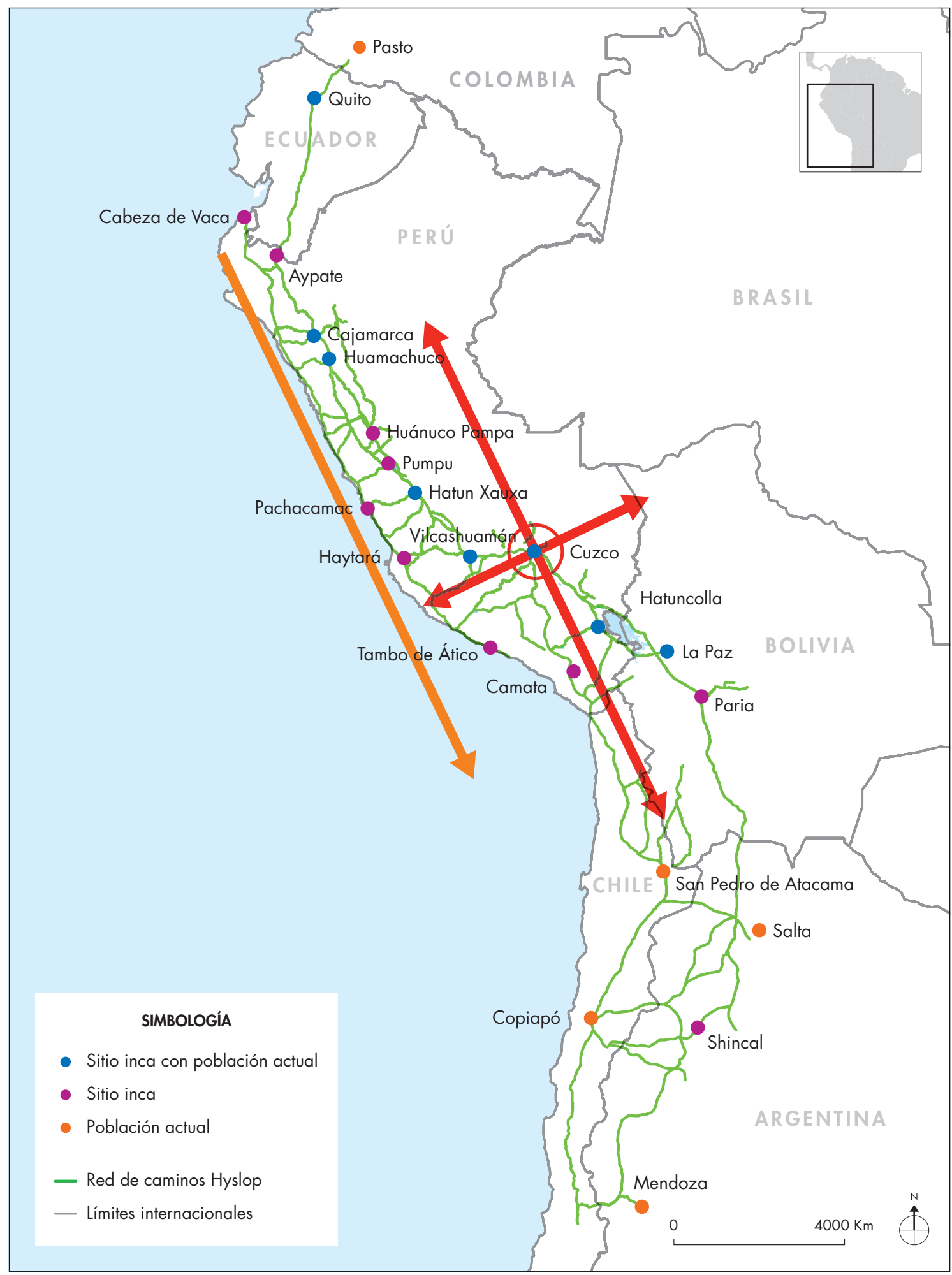

Figura 3. Mapa del Tahuantinsuyu indicando la nomenclatura de tramos según su orientación. Figure 3. Map of Tahuantinsuyu, indicating the nomenclature of road sections according to orientation. 


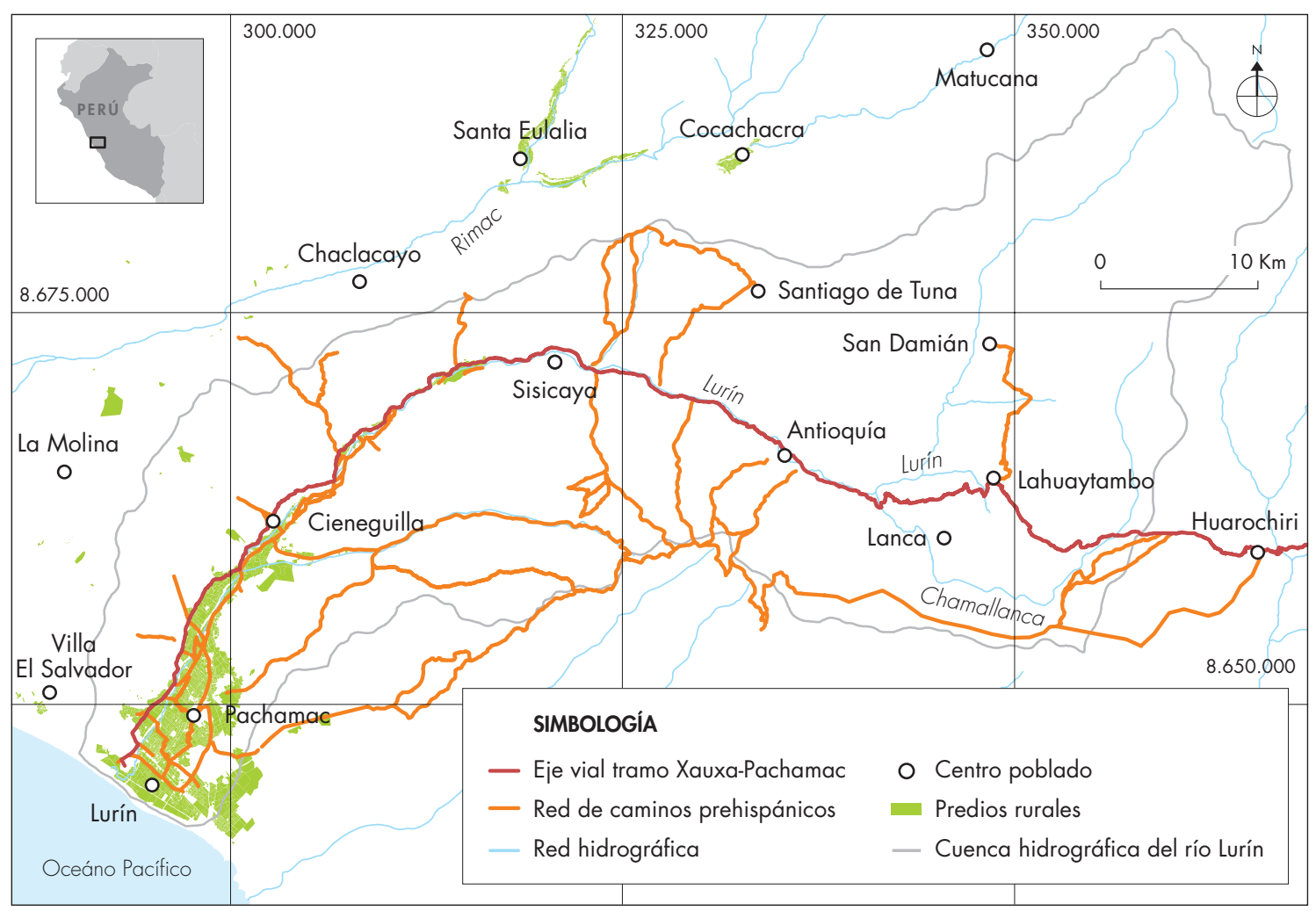

Figura 4. Red de caminos en la cuenca baja del río Lurín, Tramo Xauxa-Pachacamac (tomado de Medina 2012, redibujado por Julio Fernández 2015). Figure 4. Road network in the lower basin of the Lurin river, section Xauxa-Pachacamac (from Medina 2012, redrawn by Julio Fernández 2015.

es el Ministerio de Cultura, le ha permitido elaborar un modelo de registro que combina datos de identificación, descripción e interpretación, propias de la labor académica, con otros de cuantificación y sociabilización del quehacer técnico administrativo. Este esquema se orienta a la evaluación de aquellas secciones o segmentos que presentan evidencia arqueológica y hace una clara distinción de su particularidad física, estado de conservación, originalidad y proyección lineal, que pueden ser trasladados al Sistema de Información Geográfica (sIG) para el estudio del emplazamiento de una red especifica o los porcentajes que contrasten no solo su extensión sino también las condiciones para su defensa y aprovechamiento como recurso patrimonial, que es el propósito final del $\mathrm{PQN}-$ Sede Nacional. ${ }^{11}$

De acuerdo con las definiciones preliminares hechas por el PQÑ (2003-2008), y la Guía de identificación y registro del Qhapaq Nan, los caminos, ya sean superficies holladas debido al tránsito constante o vías construidas para el tránsito, se identifican como:
- Camino reconocido: presenta componentes estructurales bien definidos. Su calzada (la superficie acondicionada para el tránsito en el eje del camino), ya sea: empedrada, despejada o elevada, se encuentra en regular o buen estado de conservación, por lo que es posible recorrerla con relativa facilidad. Además, sus elementos de borde, como los muros (de contención o laterales) y los alineamientos de piedras, así como otros elementos integrados: puentes, canales de drenaje, cunetas, etc., son fácilmente identificables en la sección o segmento de registro.

- Trazo de camino: Define la intermitencia de evidencias o elementos estructurales del camino en contraste a la continuidad de su calzada. Un "paso natural" puede ser considerado en esta categoría en tanto exhiba en su derrotero elementos integrados como: saywas, apachetas, marcadores, etc. Aquí se encuentran también los caminos caravaneros que se extienden en los llanos de la costa y puna y se distinguen solo por su desgaste. 
- Camino afectado: presenta evidencias claramente reconocibles pero que se encuentran en mal estado de conservación debido a peligros naturales (deslizamientos de ladera, caídas de roca, flujo de lodo, etc.) o antrópicas (redes viales, expansión agrícola, predios urbanos etc.) que dificultan su recorrido. El camino no ha perdido su originalidad, sino que los factores indicados han "dañado" su estructura arquitectónica, la misma que es factible de ser recuperada. Aquí se incluyen las secciones "reconstruidas" sin supervisión técnica.

Del mismo modo, el uso de la "posible ruta" se orienta en la evaluación de los cambios sostenidos en el tiempo de aquellas secciones o segmentos que reflejan una:

- Proyección por daños: línea imaginaria que recrea la ruta del camino que ha sido destruido totalmente por causas naturales (deslizamientos de ladera o caídas de roca, flujo de detritos, etc.) o antrópicas (redes viales, expansión agrícola, predios urbanos, actividad minera, etc.); su uso facilita unir dos secciones que cuentan con clara evidencia arqueológica.

- Proyección por remplazo: línea eje de registro en la que se tiene la superposición específica de vías de transporte moderno como carreteras y caminos de herradura. No obstante, en esta puede seguirse el derrotero original del camino con base en documentos históricos que detallan su trayectoria y a la recurrencia de sitios que vinculaba. Como ejemplo, se tiene la carretera central, que en la ciudad de Jauja se ha superpuesto al camino que ingresaba a la plaza del antiguo centro administrativo inca de Hatun Xauxa. Aunque la superposición de infraestructura vial es recurrente en todo el Qhapaq Ñan (Matos 1992; Bar 2013), cabe aclarar que si una vía moderna no cuenta con suficiente respaldo histórico o arqueológico debe ser consignada en la siguiente categoría.

- Proyección por ausencia: línea eje de registro cuyo recorrido, a pesar de no contar con evidencia estructural, por las condiciones topográficas del entorno, hace posible conectar dos secciones de camino bien definidas. Muy aparte de los cortes de ladera por obras viales modernas, aquí pueden citarse las áreas de bofedal de puna o los corredores naturales formados al pie de estribaciones costeras.
Cada una de las categorías definidas puede ser representada en una serie de líneas con codificaciones de color para su reconocimiento en los softwares Google Earth o Bing Maps como soporte grafico para el diagnóstico situacional previo a la creación de una base de datos SIG en los que puedan articularse los ejes lineales y nodos del Qhapaq Nan. La figura 5 resume los criterios de codificación con uso de la escala de colores Google Earth.

En caso de que la ruta seguida no guarde relación concreta con el eje vial de un camino arqueológico, pero por temas de operatividad sea considerada en la cuantificación métrica del mismo, debe ser categorizada entonces como "trazo recorrido", detallando las razones para dicha inclusión. ${ }^{12}$ Asimismo, si por razones ajenas al trabajo de campo, extensas áreas geográficas no fueron prospectadas, pero en la revisión de imágenes satelitales, fotográficas o cartográficas (históricas o actuales) se observa su proyección, estas deben ser consignadas como tentativas o "identificadas" y deberán ser también sustentadas técnicamente.

La aplicación práctica de todo lo dicho puede verse en el diagnóstico situacional del camino prehispánico Huancas-río Utcubamba $(8,12 \mathrm{~km})$, ubicado en la región Amazonas, que fuera llevado a cabo por el PQÑ en el 2013 y derivara en su declaratoria como Patrimonio Cultural y el establecimiento de su área intangible por la Dirección de Catastro del Ministerio de Cultura el año 2014 (tabla 1).

\section{A MODO DE CONCLUSIÓN}

El estudio y el análisis de los caminos deben realizarse con la integración de múltiples capas de información que comprendan la revisión de datos provenientes de la arqueología, historia, toponimia, etnografía (tradición oral), documentación fotográfica y cartográfica, sea histórica o reciente, así como de otras fuentes relacionadas a estas que permitan reconocer las características del objeto de estudio: en nuestro caso, la red vial inca. Todo este bagaje de información debe ser incorporado a un sistema de información geográfica, que a su vez brinde un mayor conocimiento de los caminos registrados a fin de desarrollar las estrategias de investigación o de intervención. En ese sentido, las conceptualizaciones hechas nos ayudan a tener un mejor discernimiento de los caminos y de su articulación e interacción con los sitios arqueológicos, al igual que un mejor manejo 


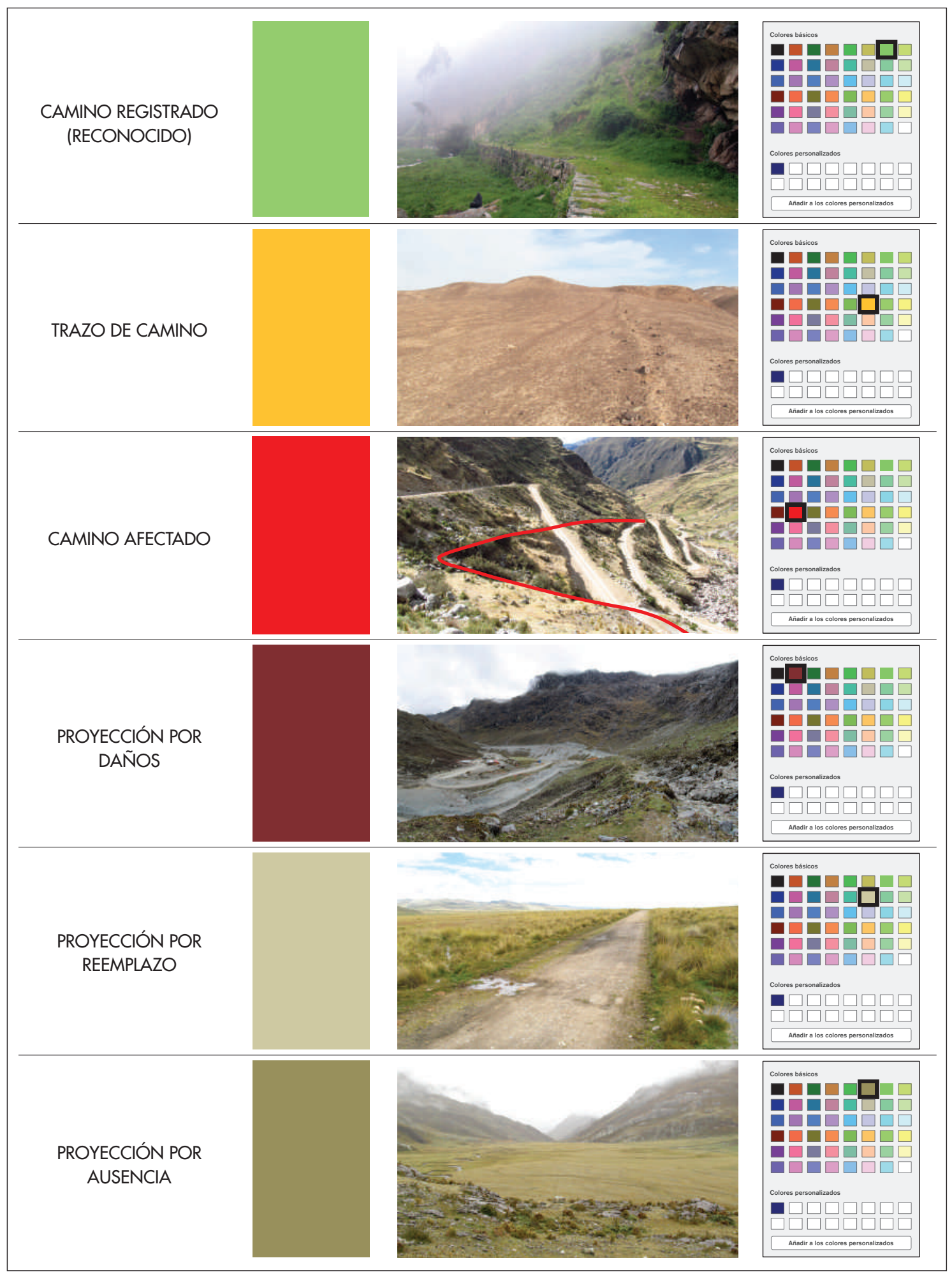

Figura 5. Categorización y codificación de color en la proyección visual de caminos. Figure 5. Categorization and color codes in the visual projection of roads. 


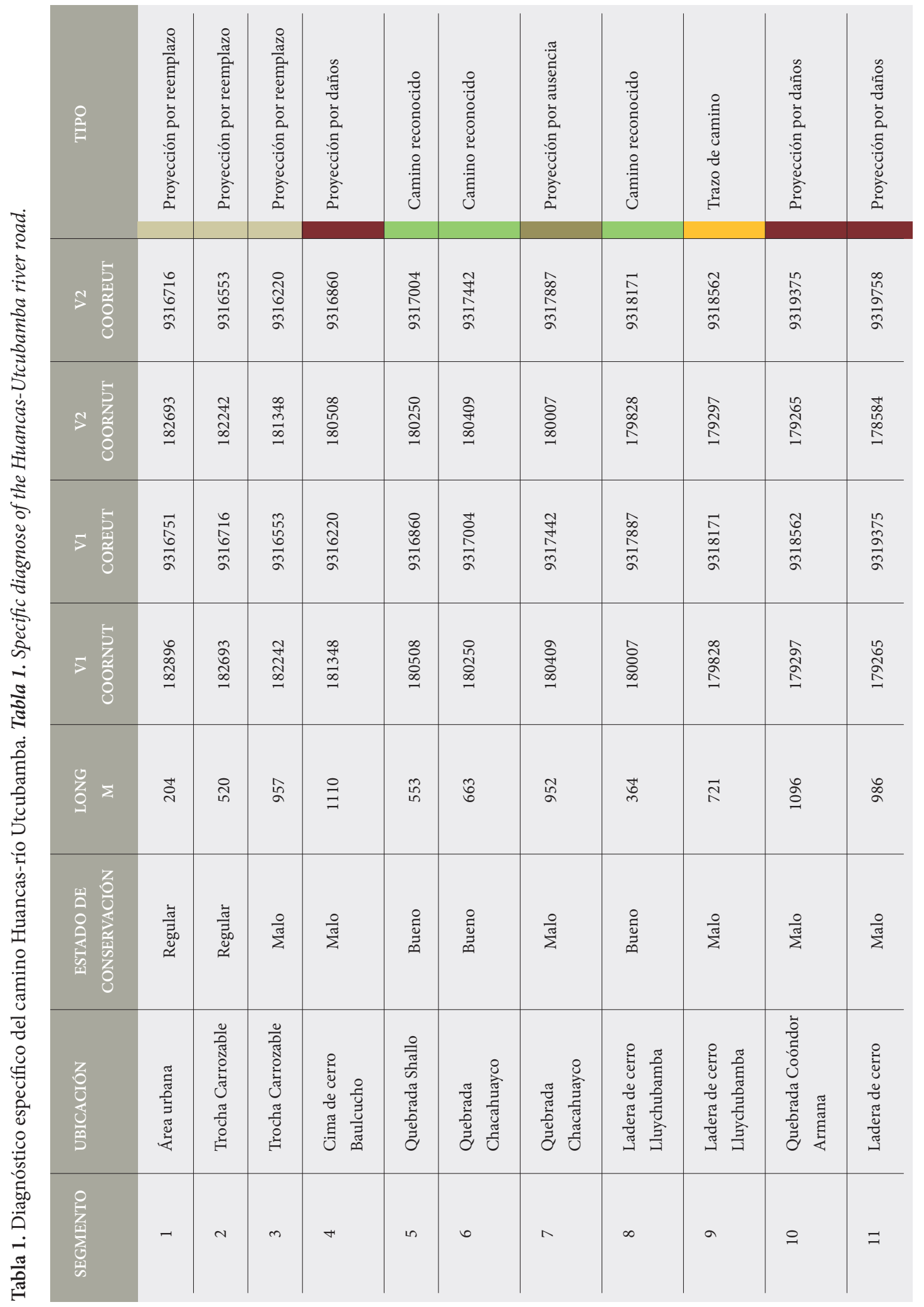


de la data misma. Además, la categorización planteada permite realizar un diagnóstico inmediato de su extensión total, en el que puede conocerse el proceso de cambio ocurrido a través del tiempo (reflejado en el historial de registro trasladado a la Geodatabase).

Lo expuesto tiene como fin estandarizar -al menos en territorio peruano- los criterios para la sectorización y nomenclatura de las diversas secciones de camino que puedan ser reconocidas como pertenecientes a la red vial inca, ya sea en el marco de un proyecto de investigación o dentro de la arqueología de contrato; sin embargo, esta propuesta puede ser revisada en el estudio de las redes viales originadas en las diferentes etapas culturales y bajo los criterios de cada realidad institucional, social o profesional, por lo que los planteamientos hechos son perfectibles.

\section{NOTAS}

${ }^{1}$ Instituto Nacional de Cultura $(2004,2006)$.

${ }^{2}$ Elaborado en la vir Reunión de Expertos del Qhapaq Nan, Pasto, Colombia 2006.

${ }^{3}$ En dichas campañas el tramo se definía como: "cada una de las partes en que está dividido o se puede dividir una extensión lineal. Los caminos se dividen en tramos los cuales son arbitrarios, pero generalmente se denominan por los asentamientos que están en sus extremos" (Álvarez \& Espinoza 2004: 23).

${ }^{4}$ Los proyectos en cuestión son: el Proyecto de evaluación arqueológica con excavaciones para la construcción de la carretera Azul Mina-Matash-Huanzalá Sur, distrito de Huallanca, Provincia de Bolognesi, Región Ancash (2007) y el Proyecto de reconocimiento arqueológico "Línea de transmisión LT-33KV S. E. Huallanca Nueva - S. E. Pucarrajo" (2008).

${ }^{5}$ Caso del camino costero en la región Tumbes. El tramo en estudio comprende desde la quebrada Cusco, en la montaña de los Amotapes, hasta Playa Hermosa en el litoral; tiene una longitud de casi $100 \mathrm{~km}$ con dirección de sur a norte, con alturas que oscilan entre 1538 y 0 msnm. Políticamente se ubica entre los distritos de San Jacinto y Corrales, Provincia y Departamento de Tumbes (Vilches 2013: 4).

${ }^{6}$ Estas localidades son también nodos del Camino Longitudinal de la Sierra y el Camino de los Llanos o Longitudinal de la Costa.

${ }^{7}$ La diferenciación de nodos se hace por el carácter funcional y jerárquico de los sitios que representan: los nodos primarios corresponden a los sitios (de filiación inca) que definen el origen y extensión de un eje vial; los nodos secundarios a los sitios que se ubican al interior del mismo y que sirven como controladores del flujo; y los nodos de menor rango se relacionan con todos los sitios -de indistinta filiación- que son interconectados por el eje vial o los caminos que se desprenden de este.

${ }^{8}$ Su identificación se dio dentro del Proyecto de investigación y registro con excavaciones en la red de caminos inca en la meseta de Bombón, dirigido por el autor el presente año.

${ }^{9}$ Esta relación debe primar sobre las descripciones halladas en fuentes etnohistóricas para no crear definiciones forzadas (véase Barraza 2010; Chacaltana 2010).

${ }^{10}$ Algunas veces con evidencia de origen y función militar, como en el caso de Inkahuasi de Lunahuaná, que fue construido como un "nuevo Cusco" durante el tiempo que duró el conflicto bélico entre el ejército cusqueño y las fuerzas del señorío de Guarco (Hyslop 1985).

${ }^{11}$ Toda esta información es llevada a una Geodatabase que puede ser visualizada en el Sistema de Información Geográfica de Arqueología (SIGDA) del Ministerio de Cultura.

${ }^{12}$ Esta situación había sido ya prevista el año 2005, por lo que en documentos internos del PQÑ el "recorrido" representa el espacio caminado siguiendo una determinada ruta sin encontrar evidencia del camino. Sin embargo, en el registro del año 2008 no fue consignado como una categoría en sí misma, por lo que en ocasiones fue incluido como parte de la "posible ruta".

\section{REFERENCIAS}

Acosta, J., 1954 [1590]. Historia natural y moral de las Indias. Biblioteca de Autores Españoles, Tomo 73. Madrid: Atlas. Álvarez, A. \& J. Espinoza., 2004. Glosario de tecnología caminera. Documento de trabajo del componente de arqueología del Proyecto de levantamiento del sistema vial inca. Proyecto Qhapaq Nan-INc. Lima.

Astuhuamán, C., 2010. La red de sitios y Caminos Incas en la sierra de Piura, Perú. Inka Llaqta 1: 29-60. Lima.

BÁKulA, C., 2009. Qhapaq Nan, trabajo permanente. Gaceta Cultural del Perú 38: 4-5. Lima: Instituto Nacional de Cultura.

BAR, A., 2008 Ms. Actividad de identificación y registro del sistema vial inca. Macro Región Centro, Ancash-Huánuco. Informe final, Proyecto Qhapaq Ñan. Lima: Instituto Nacional de Cultura.

BAR, A., 2013. Afectaciones históricas a la red vial inca y la necesidad del estudio documentario de carreteras para la investigación y registro de caminos prehispánicos. Cuadernos del Qhapaq Nan 1: 169-179. Lima: Ministerio de Cultura.

BARRAZA, S., 2010. Redefiniendo una categoría arquitectónica inca: la kallanka. Boletín del Instituto Francés de Estudios Andinos 39 (1): 167-181. Lima.

Bernabé, J., 2008 Ms. Actividad de identificación y registro del sistema vial inca. Macro Región Centro, Junín-Huancavelica. 
Informe final, Proyecto Qhapaq Ñan. Lima: Instituto Nacional de Cultura.

Casaverde, G. \& S. López, 2010. Principios metodológicos para la identificación y registro arqueológico de los Caminos Inka. Inka Llaqta 1: 79-102. Lima.

Castro, V.; V. Varela, C. Aldunate \& E. Araneda, 2004. Principios orientadores y metodología para el estudio del Qhapaqñan en Atacama: desde El Portezuelo del Inka hasta Río Grande. Chungara 2 (36): 463-481.

Chacaltana, S., 2010. Evidencias arqueológicas en Camata Tambo, tambo inca ubicado en el valle alto de Moquegua, Andes sur-centrales. Arqueología y sociedad 21: 145-170. Lima.

Cieza, P., 1995 [1553]. Crónica del Perú. Primera parte. Lima: Fondo Editorial de la Pontificia Universidad Católica del Perú-Academia Nacional de la Historia.

Cieza, P., 1996 [1553]. Crónica del Perú. Segunda parte. Lima: Fondo Editorial de la Pontificia Universidad Católica del Perú-Academia Nacional de la Historia.

Estete, M., 1891 [1533]. La relación del viaje que hizo el señor capitán Hernando Pizarro por mandado del señor gobernador, su hermano, desde el pueblo de Caxamalca á Parcama, y de allí á Jauja. En Verdadera relación de la Conquista del Perú y provincia del Cuzco, F. Xerez, pp. 119149. Madrid: Establecimiento tipográfico de J. C. García.

FigueroA, L., 2009. Un legado que articula pasado y presente. Camino a patrimonio de la Humanidad. Gaceta Cultural del Perú 38: 10-11. Lima: Instituto Nacional de Cultura.

Gasparini, G. \& L. Margolies, 1977. Arquitectura inca. Caracas: Universidad Central de Venezuela, Centro de Investigaciones Históricas y Estéticas. Facultad de Arquitectura y Urbanismo.

Garcilaso de la Vega, I., 2007 [1604]. Comentarios reales de los Incas. Lima: Fondo Editorial Universidad Inca Garcilaso de la Vega.

Guaman Poma, F., 2008 [1615]. Nueva corónica y buen gobierno. Lima: Fondo de Cultura Económica.

Hagen, V., 1977. La carretera del Sol, la búsqueda del camino de los incas. México D.F.: Diana.

Instituto Nacional de Cultura, 2006. Programa Qhapaq Ñan: informe por Cuencas Hidrográficas del Registro de Tramos y Sitios. Campañas 2003-2004. Lima.

Instituto Nacional de Cultura, 2005. Proyecto Qhapaq Ñan-Macro Región Centro. Campañas 2003-2004. AncashLima-Huánuco-Pasco-Junín. Dirección de Arqueología. Documento digital de trabajo del Proyecto Qhapaq Ñan (CD). Lima.

Instituto Nacional de Cultura, 2004. Proyecto Qhapaq Ñan: Informe de Campaña 2002-2003. Lima.

Hyslop, J., 1985. Incahuasi the new Cuzco. Cañete, Lunahuaná, Perú. Nueva York: Institute of Andean Research.

Hyslop, J., 1992. Qhapaq Nan. El sistema vial incaico. Lima: Instituto Andino de Estudios Arqueológicos INDEAPetróleos del Perú.
Hyslop, J., 2004. Proyecto Qhapaq Nan: Informe de Campaña 2002-2003. Lima.

Hyslop, J., 2005. Proyecto Qhapaq Nan-Macro Región Centro. Campañas 2003-2004. Ancash-Lima-Huánuco-Pasco-Junín. Dirección de Arqueología. Documento digital de trabajo del Proyecto Qhapaq Nan (CD). Lima.

Kendall, A. 1976. Descripción e inventario de las formas arquitectónicas inca. Revista del Museo Nacional 42: 13-96. Lima.

Matos, R., 1992. El Camino Real Inca y la carretera moderna en Chinchaycocha, Junín. En Estudios de arqueología peruana, D. Bonavia \& M. Biggar, Eds., pp. 375-392. Lima: FOMCIENCIAS.

Medina, G., 2012 Ms. Análisis de la información medioambiental de la red de caminos en el Subtramo PachacamacAntioquia. Informe parcial, Proyecto Qhapaq Nan, Ministerio de Cultura. Lima.

Middendorf, E., 1973-1974 [1893-1895]. Perú, Observaciones y estudios del país y sus habitantes durante una permanencia de 25 años. Tomos 1-3. Lima: Universidad Nacional Mayor de San Marcos.

Ministerio de Cultura, 2013. Guía de identificación y registro del Qhapaq Ñan. Lima: Ministerio de Cultura.

Murúa, M., 2001 [1590]. Historia general del Perú. Madrid: Dastin.

Raffino, R., 1981. Los Inkas del Kollasuyo. La Plata: Ramos Americana Editora.

Raimondi, A., 1874. El Perú, Tomo I. Lima: Imprenta del Estado. RAIMONDI, A., 1875. El Perú, Tomo II. Lima: Imprenta del Estado.

Raimondi, A., 1894. El Perú, Tomo III. Lima: Imprenta del Estado.

Regal A., 1936. Los Caminos del Inca en el Antiguo Perú. Lima: Sanmartí.

Regal A., 1972. Los puentes del Inca en el antiguo Perú. Lima: Imprenta Gráfica Industrial.

Ríos, S., 2014. Los proyectos arqueológicos desarrollados entre los años 2003 y 2009 como insumo para la identificación y registro del Qhapaq Ñan. Cuadernos del Qhapaq Nan 2: 169-179. Lima: Ministerio de Cultura.

Sarmiento de GAmboA, P., 2001 [1572]. Historia de los Incas. Madrid: Miraguano.

Strube, L. 1963. Vialidad imperial de los Incas. Desde Colombia hacia Chile central y sur de Mendoza (Argentina) con inclusión de sus proyecciones orientales. Córdoba: Universidad Nacional de Córdoba, Instituto de Estudios Americanistas, serie Histórica xxxiII.

Topic, J., \& T. L. Topic, 2014. Relaciones costa-sierra en el norte del Perú: algunas observaciones sobre rutas, redes y escalas de interacción. Cuadernos del Qhapaq Nan 2: 50-67. Lima: Ministerio de Cultura.

Tschudi, J., 1966 [1846]. Testimonio del Perú, 1838-1842. Lima: Talleres Gráficos P. L. Villanueva.

Vaca de Castro, C., 1909 [1543]. Ordenanza de tambos. Histórica 3: 427-492. Lima. 
VArela, F., 2003 Ms. Macro Región Centro, Tramo Acostambo (Huancavelica)-Huamachuco (La Libertad). Informe final, Proyecto Qhapaq Ñan. Lima: Instituto Nacional de Cultura. Vílchez, C., 2013. El Camino Inca de la costa en Tumbes. $<$ http://qhapaqnan.cultura.pe/sites/default/files/articulos/2. pdf $>$ [Consultado 13-09-17].
VITRY, C., 2007. Caminos rituales y montañas sagradas. Estudio de la vialidad inka en el nevado de Chañi, Argentina. Boletín del Museo Chileno de Arte Precolombino 12 (2): 69-84, Santiago de Chile.

Wiener, C. 1993 [1880]. Perú y Bolivia: relato de viaje. Lima: Instituto Francés de Estudios Andinos-Universidad Nacional Mayor de San Marcos. 